\title{
A Case Series of COVID-19 Respiratory Infection in Hospitalized Children: Clinical and Computed Tomography Challenges
}

\author{
Fatemeh Zamani (iD ${ }^{1,}{ }^{*}$, Afshar Ghamari ${ }^{2}$, Gisoo Darban Hosseini Amirkhiz ${ }^{3}$, Neda Pak ${ }^{1}$ and Omid \\ Ghaemi $^{1}$ \\ ${ }^{1}$ Department of Radiology, Tehran University of Medical Sciences, Tehran, Iran \\ ${ }^{2}$ Department of Radiology, Faculty of Medicine, Mashhad University of Medical Sciences, Mashhad, Iran \\ ${ }^{3}$ Research Center for Prevention of Cardiovascular Disease, Institute of Endocrinology and Metabolism, Iran University of Medical Sciences (IUMS), Tehran, Iran \\ "Corresponding author: Department of Radiology, Tehran University of Medical Sciences, No. 14, South Golestan Alley, Movahed Danesh St, Tehran, Iran. Email: \\ doctorzamani@gmail.com
}

Received 2020 June 22; Revised 2021 February 01; Accepted 2021 February 13.

\begin{abstract}
Our objective was to evaluate clinical and computed tomographic features in hospitalized children with coronavirus disease 2019 (COVID-19) infection who were admitted to the pediatric center of excellence, children's medical center. In this case-series, we report twelve patients with clinical symptoms of acute respiratory infections at the beginning or after admission who have polymerase chain reaction (PCR)-proved (throat swab samples) COVID-19 infection and underwent computed tomography imaging. Six patients had an underlying disease and atypical findings with no clinical suspicion at the beginning. Tachypnea (75\%) represented the most common physical finding. Computed tomography was abnormal in 83.3\% of patients. Subpleural consolidation, peribronchial thickening, round consolidation, and Halo sign was depicted. The empirical regimen in our hospital included hydroxychloroquine and oseltamivir. Some of the patients managed easily, but others had prolonged hospital stay, especially patients with significant underlying conditions.
\end{abstract}

Keywords: COVID-19, Pediatric, Computed Tomography, Clinical Features

\section{Introduction}

On 31 December 2019, an unknown viral pneumonia was reported from Wuhan, China. The causative virus was found to be a new coronavirus. On 11 February 2020, the World Health Organization (WHO) officially named the disease caused by this organism as coronavirus disease of 2019 (COVID-19). WHO declared the infection as a global health emergency on January 30, 2020 (1).

The susceptible population are mainly adults and those with a suppressed immune system. The infectious rate of COVID-19 is relatively lower in children (2). A review by the Chinese Center for Disease Control and Prevention demonstrated that among 72,314 cases, less than $1 \%$ were children younger than 10 years old (3). The first reported case was a 7-year-old boy with cough and fever confirmed on 19 January 2020 (4). In the pediatric population, the symptoms were initially reported to be absent or mild, but with an increasing number of affected patients more symptomatic children are being encountered $(5,6)$.

Previously published data are mainly from China, but less than $10 \%$ of confirmed cases are now from this country. This emphasizes the need for more data from other parts of the world to discover the potential hidden aspects of the disease in the pediatric population.

This study was conducted in a one-month period from 1 March 2020 until 1 April 2020 according to relevant guidelines and regulations in the Pediatric Center of Excellence, Tehran University of Medical Sciences, Tehran, Iran. We report these pediatric patients with typical and atypical presentation of COVID-19 infection and heterogeneous unexpected features in their imaging.

All patients were under 18 years old with clinical symptoms of acute respiratory infections at the beginning or after admission who underwent computed tomography imaging and had confirmed COVID-19 infection (nucleic acid test, throat swab samples). We reviewed the clinical assessments, laboratory and imaging findings of these pa- 
tients from electronic records. It should be noted that all patients had a chest radiograph (CXR) before computed tomography (CT) scan examination. All images were reviewed by a pediatric radiologist. CT findings were reported and discrepancies were tackled via discussion.

\section{Case Presentation}

\subsection{Cases 1 and 2}

These two 9-year-old patients were monozygotic twin boys (Table 1). They did not have any underlying diseases. They had continuous exposure to their infected grandfather. Their clinical presentation was atypical. They suffered fever, headache, nausea, vomiting, and diarrhea. They did not have any respiratory symptoms. Laboratory data revealed leukopenia and positive reverse transcription polymerase chain reaction (RT-PCR). Elevated Creactive protein (CRP) was detected in one of them (Table 2). The chest computed tomography findings were similar ground-glass opacities in lower lobes in both patients (Figure 1). They received supportive care, antiviral drugs, and appropriate antibiotics. They were finally discharged from the hospital after six days, without need for ICU admission.

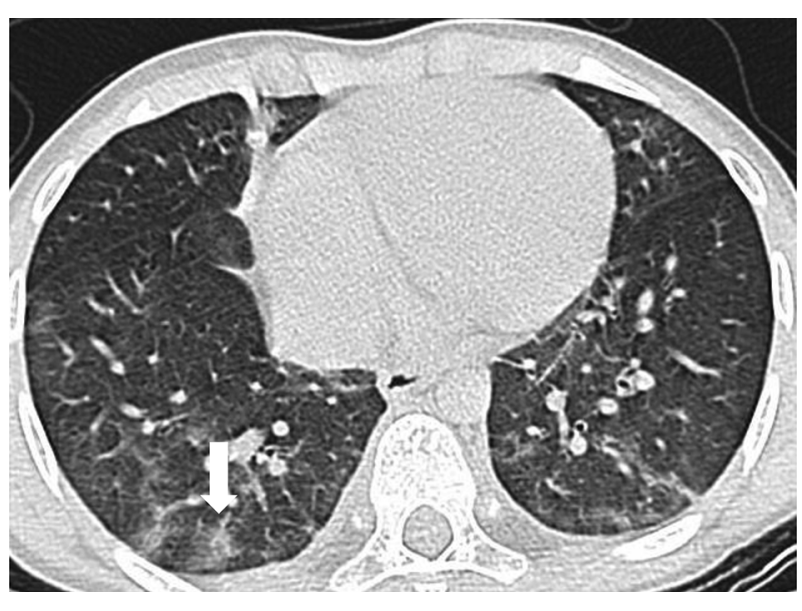

Figure 1. Axial chest CT scan in a 9-year-old boy with COVID-19 infection shows subpleural alveolar consolidations in lower lobes (white arrow) (patient no. 1).

\subsection{Case 3}

An 11-year-old cirrhotic girl in whom epistaxis was preceding respiratory symptoms (Table 1 ). She did not experience any close contact with an infected person. The peribronchial thickening and diffuse reticular pattern were seen in CXR. Ground glass opacities (GGO) and sub-pleural consolidation were evident in the chest CT scan. Her management protocol is summarized in Table 3. She was discharged after 6 days.

\subsection{Cases 4, 5, 6, and 9}

These cases had no underlying disease. Two of them (patients 6 and 9) had household contact with a COVID19 infected person. Most of them had fever and cough. Three of them (patients 5, 6, and 9) suffered abdominal pain. Their RT-PCR test results were positive. In chest CT collapse-consolidation in lower lobes in patient 4, patchy ground glass opacities in both lobes and interlobular septal thickening in lower lobes in patient 5 , solid nodules with peripheral ground glass opacities in the right lower lobe in patient 9 were seen. Chest CT of patient 6 was normal. Their management protocol was based on supportive care and empiric regimen. For the 6-year-old girl, cefotaxime and vancomycin were added to our hospital empirical regimen. All patients were treated successfully.

\subsection{Case 7}

This case was an 11-year-old boy with heart failure who presented with fever resistance to the standard treatment. Other symptoms are mentioned in Table 1. CXR and chest CT scan were normal, but he had a positive RT-PCR of COVID-19. Laboratory tests are shown in Table 2. He was managed with an empirical regimen after 6 days.

\subsection{Case 8}

A 6-year-old boy with newly diagnosed Wilson's disease came with a comprehensive set of abdominal symptoms for one week. His presenting symptoms were as follows: mild umbilical abdominal pain, one episode of bloody vomiting, mildly decreased urine output, and progressive abdominal distension. One day before admission, fever, rhinorrhea, respiratory distress, and cough were added to his symptoms. Bilateral pleural effusion and alveolar consolidation in the right lower lobe were detected in chest CT. His management and treatment are illustrated in Table 3. He was discharged after 19 days of hospital admission.

\subsection{Case 10}

She was a known case of acute lymphoblastic leukemia (ALL) for 3 years who underwent human stem cell transplantation 19 days ago. She had no close contact with an infected person. Her presentation symptoms were fever, 


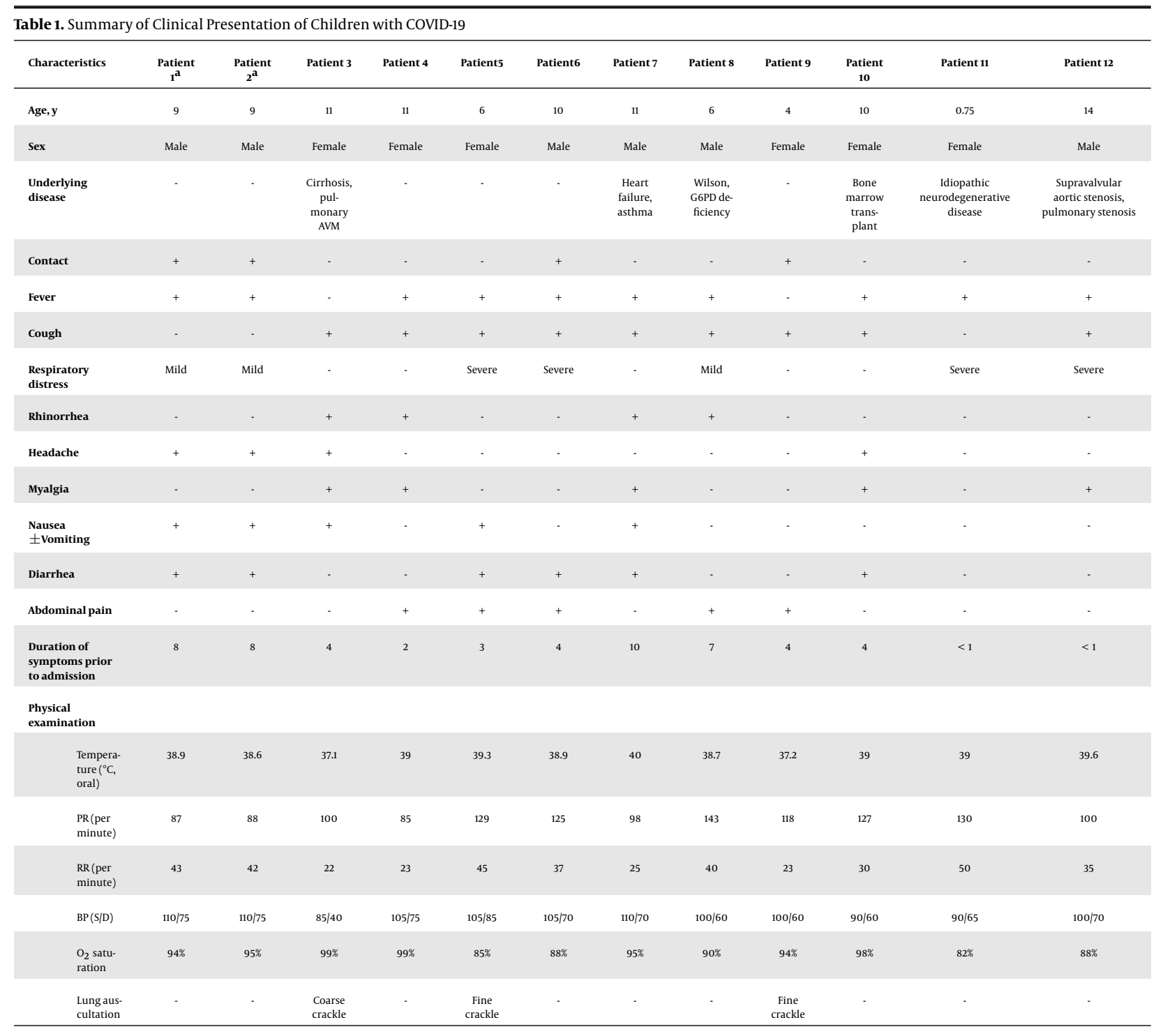

Abbreviations: AVM, arteriovenous malformation; BP, blood pressure; COVID-19, corona virus disease 2019; G6PD, glucose-6-phosphate dehydrogenase; RR, respiratory rate; PR, Pulse rate; S/D, systolic/diastolic ${ }^{\mathrm{a}}$ Monozygotic twin.

\begin{tabular}{|c|c|c|c|c|c|c|c|c|c|c|c|c|}
\hline Characteristics & Patient $1^{\mathrm{a}}$ & Patien $\mathbf{t ~}^{\mathrm{a}}$ & Patient 3 & Patient 4 & Patien $t 5$ & Patien 16 & Patient 7 & Patient 8 & Patient 9 & Patient 10 & Patient 11 & Patient 12 \\
\hline \multicolumn{13}{|l|}{ Laboratory test } \\
\hline $\mathrm{WBC}, \times 10^{3} / \mu \mathrm{L}$ & 2.38 & 2.56 & 1.38 & 5.6 & 3.11 & 1.9 & 3.65 & 7.4 & 11.09 & 3.52 & 9.21 & 12.89 \\
\hline Lymphocyte,\% & 25 & 17 & 36 & 25 & 16 & 15 & 52 & 48.7 & 26 & 13 & 52 & 7 \\
\hline PLT, $\times 10^{3} \mid \mu \mathrm{L}$ & 201 & 253 & 68 & 237 & 521 & 110 & 273 & 91 & 339 & 211 & 159 & 41 \\
\hline CRP, $\mathrm{mg} / \mathrm{L}$ & 5 & 13 & 5 & 34 & 49 & 1 & 5 & 9 & 2 & 1 & 50 & 520 \\
\hline ESR, $\mathbf{m m} / \mathbf{h}$ & 1 & 22 & 1 & 22 & 25 & 3 & 20 & 12 & 30 & 13 & 26 & 56 \\
\hline
\end{tabular}

Abbreviations: AVM, arteriovenous malformation; COVID-19; corona virus disease 2019, CRP, C-reactive protein; ESR, erythrocyte sedimentation ratio; G6PD, glucose-6-phosphate dehydrogenase; WBC, white blood cell. a Monozygotic twin. 


\begin{tabular}{|c|c|c|c|c|c|c|c|c|c|c|c|c|}
\hline Characteristics & Patient $\mathbf{~}^{\mathrm{a}}$ & Patient $\mathbf{2}^{\mathrm{a}}$ & Patient 3 & Patient 4 & Patient 5 & Patient 6 & Patient 7 & Patient 8 & Patient 9 & Patinet 10 & Patient 11 & Patient 12 \\
\hline Treatment & $\begin{array}{l}\text { Hcq, Oselta, } \\
\text { Cfm, Amp }\end{array}$ & $\begin{array}{l}\text { Hcq, Oselta, } \\
\text { Cfm, Amp }\end{array}$ & $\begin{array}{l}\text { Hcq, Oselta, } \\
\text { Azt }\end{array}$ & $\begin{array}{l}\text { Hcq, Oselta, } \\
\text { Azt }\end{array}$ & $\begin{array}{l}\text { Hcq, Oselta, } \\
\text { Cfm, Vanco }\end{array}$ & $\begin{array}{l}\text { Hcq, Oselta, } \\
\text { Azt }\end{array}$ & Oselta, Azt & $\begin{array}{l}\text { Hcq, Cfm, } \\
\text { Met }\end{array}$ & $\begin{array}{l}\text { Hcq, Oselta, } \\
\text { Azt }\end{array}$ & $\begin{array}{l}\text { Hcq, Azt, } \\
\text { Vor, Mer }\end{array}$ & $\begin{array}{l}\text { Hcq, Oselta, } \\
\text { Azt, Vanco, } \\
\text { Mer }\end{array}$ & $\begin{array}{l}\text { Hcq, Oselta, } \\
\text { Azt, Cef, } \\
\text { Levo, Vanco }\end{array}$ \\
\hline $\mathrm{O}_{2}$ therapy & Nasal & Nasal & $\mathrm{N} / \mathrm{A}$ & $\mathrm{N} / \mathrm{A}$ & Mask & Mask & $\mathrm{N} / \mathrm{A}$ & Nasal & $\mathrm{N} / \mathrm{A}$ & $\mathrm{N} / \mathrm{A}$ & $\begin{array}{l}\text { Mechanical } \\
\text { ventilation }\end{array}$ & Mask \\
\hline $\begin{array}{l}\text { ICU } \\
\text { admission }\end{array}$ & - & - & - & - & + & . & . & - & - & . & + & - \\
\hline $\begin{array}{l}\text { Hospital } \\
\text { stay, d }\end{array}$ & 6 & 6 & 6 & 6 & 10 & 3 & 6 & 19 & 4 & 4 & $13 \mathrm{ND}$ & 13 \\
\hline
\end{tabular}

cough, and diarrhea. The patchy alveolar consolidations with peripheral ground glass halo in the right lung and left lower lobe were seen in her chest CT. One antifungal agent was also added to her empirical regimen.

\subsection{Case 11}

She was a 9-month-old floppy baby. She was admitted because of poor feeding, fever, respiratory distress, and low oxygen saturation. In her chest CT, collapse-consolidation in the apical segment of the right upper lobe with secondary elevated right diaphragm and reticular pattern in the left lung were detected. She critically needed intensive care and mechanical ventilation. She died after 13 days.

\subsection{Case 12}

This patient was a 14-year-old boy with multiple congenital heart anomalies (suspicious of William's syndrome) who was on the waiting list for Brom's surgery. In the beginning, he had squeezing, chest pain, severe respiratory distress, left lower limb claudication, and left upper limb pain for about 24 hours. There were no other clinical findings in favor of COVID-19 infection. He was initially admitted to the cardiac ward. Troponin I and creatinine phosphokinase-MB tests were normal. White blood cell (WBC) count showed mild leukocytosis (11200) without lymphopenia. Fever and non-productive cough initiated the day after admission. There were positive results for RT-PCR of COVID-19 and blood culture (staphylococcus aureus). A new complete blood test typically revealed lymphopenia $(7 \%, 902)$. Both erythrocyte sedimentation rate (ESR) and CRP were elevated. Chest CT scan findings were right pleural effusion, crazy paving and sub-pleural alveolar consolidation in the left lower lobe. After one week of proper treatment, fever and laboratory abnormalities resolved.

\section{Discussion}

In spite of the expulsive growing numbers of COVID19 patients worldwide, there are few reported cases of pediatric patients outside China $(7,8)$. Clinical findings of COVID-19 infection are poorly established in children. Our results of a detailed assessment of hospitalized patients revealed new medical scenarios and established features more in otherwise healthy children.

These twelve pediatric cases have positive RT-PCR results for COVID-19 infection. There were six (50\%) male and six (50\%) female patients. The median age was 8.47 (interquartile range [IQR] $0.75-14$ ) years. Two of the patients were monozygotic twin boys. Six patients had an underlying disease. Four patients had a positive history of close contact with an affected family member (Table 1 ).

The most common presenting symptoms were fever (83.3\%) and cough (75\%) similar to adult reports. Other symptoms including headache and myalgia were less commonly seen. We think this is because these are more subjective symptoms, difficult to be noticed by caretakers, and children were not able to articulate them. In our cases, gastrointestinal (GI) symptoms including abdominal discomfort, nausea, vomiting and diarrhea were seen in seven (58.3\%) patients. This is much more frequent than what is reported in adults. Epistaxis (patient no. 3), direct hyperbilirubinemia, ascites, abdominal distension (patient no. 8), abdominal pain (patient no. 4), poor feeding, and floppy baby (patient no. 11) were unusual presentations. Tachypnea (75\%) was the most common physical finding. Hypoxemia at the presentation was seen in five patients (41.6\%). Four patients had tachycardia (33.3\%). The duration of presenting symptoms prior to admission ranged from less than 24 hours to 10 days (mean, 4.33 days).

Leukopenia and lymphopenia were found in seven (58.3\%) and eight (66.6\%) patients, respectively. Two patients had leukocytosis (16.6\%). Elevated serum CRP and 
ESR levels were seen in five (41.6\%) and eight (66.6\%) patients, respectively. Our laboratory abnormalities were concurring with previous results in the literature $(9,10)$.

Chest CT is now considered as a key diagnostic feature of COVID-19 and plays an important role in local guidelines for prompt diagnosis $(11,12)$. Computed tomography was abnormal in 10 (83.3\%) patients. Two patients had normal chest CT scan (patients no. 6 with severe respiratory distress and patient no. 7 with underlying heart failure). Subpleural consolidation depicted in $80 \%$ of positive images (Figure 1). Multiple bilateral patchy ground-glass opacity and consolidation were seen in the $70 \%$ patients with positive chest CT (Figure 2). Interestingly, another frequent finding was peribronchial thickening which was evident in five patients with underlying disease (patients no. 3, 8, 10,11 , and 12). In one patient, solid nodules with ground glass halo were limited to the right lower lobe (Patient no. 9) (Figure 3). Round consolidation was seen in two (16.6\%) patients (Figure 4). Halo sign was seen in two cases (patients no. 9 and 10). Halo sign was not as common as mentioned in the previous studies (13). Crazy paving pattern was seen in one patient. Bilateral pleural effusion was seen in three patients with underlying disease (patients no. 8,11 and 12). Cavitation, discrete nodule and lymphadenopathy were not seen in any of the patients. Uncommon imaging findings were mostly seen in patients with underlying disease.

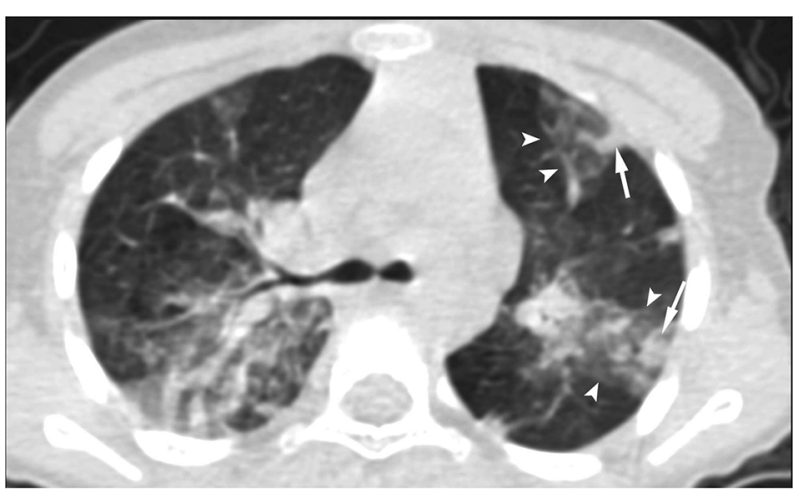

Figure 2. Axial chest CT scan in an 11-year-old cirrhotic girl shows multiple areas of consolidation (arrows) and ground-glass opacity (arrowheads) (patient no. 3).

The posterior lung was most commonly involved. In five (41.6\%) patients, lung lesions involved all five lung lobes. In two patients four lobes were involved and in two other patients, lesions were limited to both lower lobes. This concurred with previous studies which showed lower

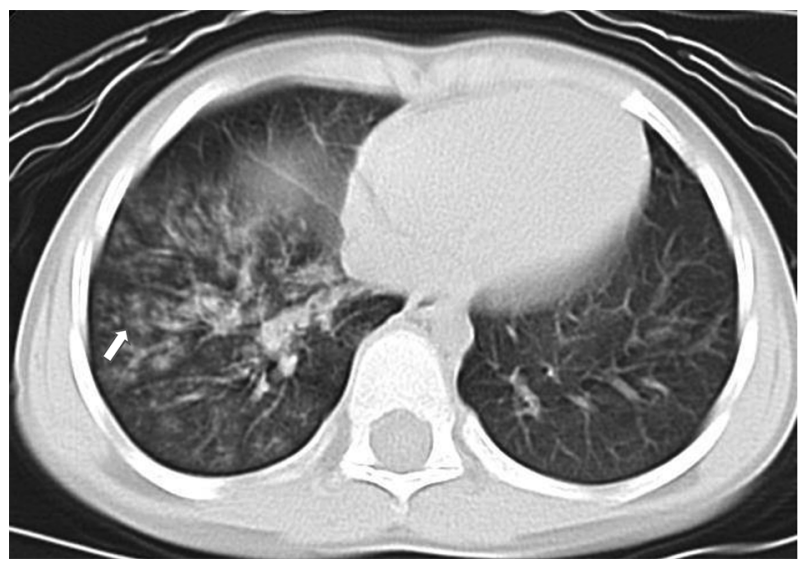

Figure 3. Axial chest CT scan in a 4-year-old girl with COVID-19 infection shows multiple solid nodules with ground glass halo in the right lower lobe (white arrow) (patient no. 9). This finding is called halo sign and has been reported in some cases of COVID-19 infection.

lobes were the most common sites of involvement (11). We also found one case with unilateral right lower lobe involvement which was also discovered in a previous investigation of COVID-19 pediatric patients (13). A prospective analysis in Wuhan revealed bilateral lung opacities in $98 \%$ of patients and described lobular and sub-segmental consolidation as the most typical finding.

The underlying disease could also change the imaging appearance. Pleural effusion and cardiomegaly, as unlikely findings, were seen in our patients. Peribronchial thickening was also a very common feature in these complex cases. This was also found in the minority of young patients during H1N1 pandemic and attributed to age related immune response (14). Like our cases, pediatric patients with the typical clinical scenario, laboratory findings and positive RT-PCR result may show a normal CT scan evaluation. A study of 171 confirmed pediatric cases showed that $27(15.8 \%)$ patients had no symptoms or imaging findings. Besides, 12 cases had radiologic findings of pneumonia with no clinical symptoms (15).

The empirical regimen in our hospital included hydroxychloroquine and oseltamivir. Due to clinical conditions and underlying disease, different antibiotics were included. In our immunocompromised patient (bone marrow transplant) one antifungal agent was also added. Hospital stay ranged from 3 to 19 days (mean, 8 days). Generally, hospital stay was longer in patients with an underlying disease. Three of our patients needed nasal oxygen therapy and the other three patients managed with the mask. 

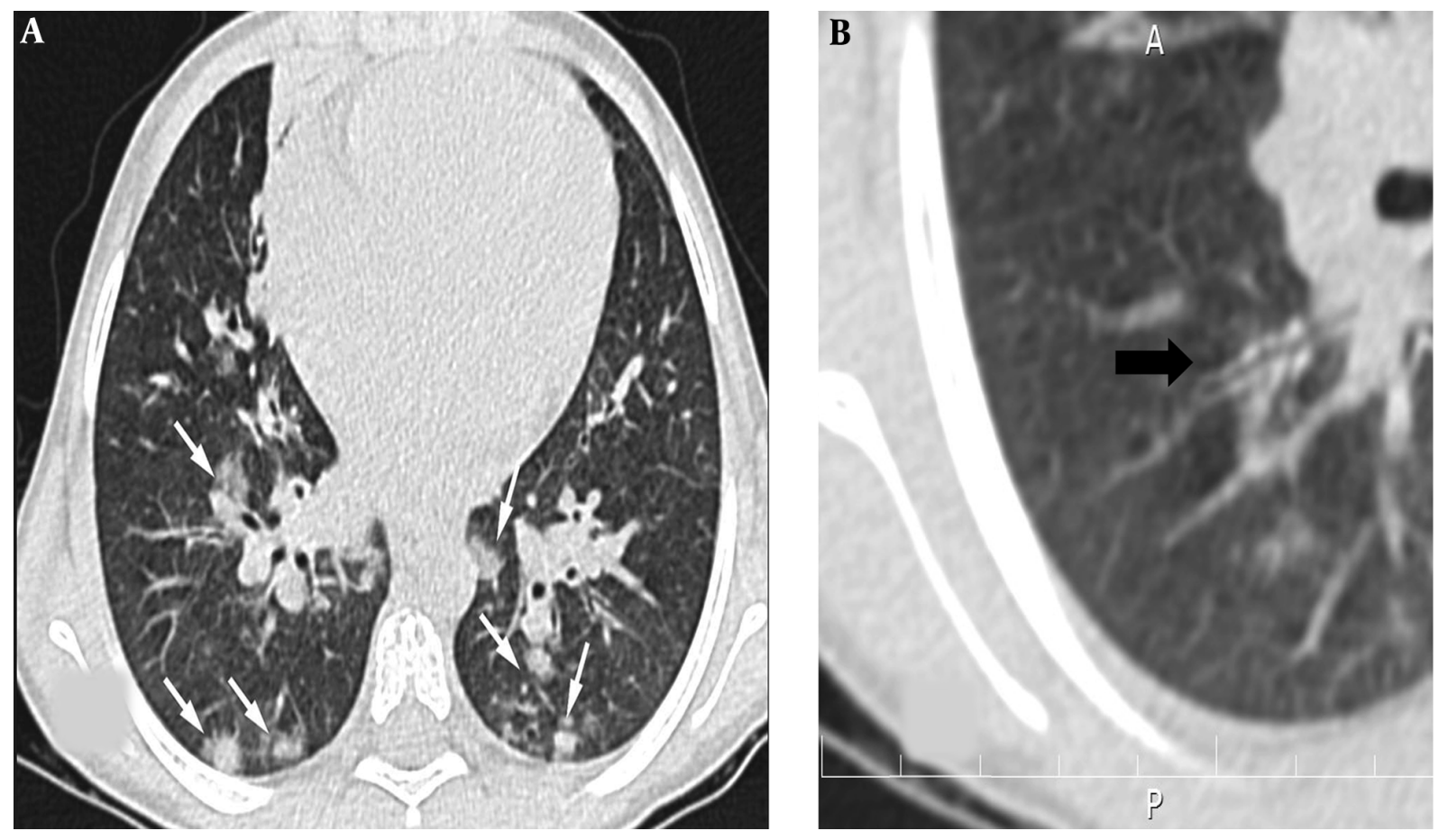

Figure 4. Axial chest CT scan in a 6-year-old girl with COVID-19 infection (patient no. 5) shows A, Multiple bilateral peripheral consolidations (white arrows) B, Peribronchia thickening (black arrow).

Mechanical ventilation was applied to one patient. Complete resolution of symptoms, stable vital signs with normal WBC and lymphocyte counts, and normal level of CRP and ESR, along with negative repeated RT-PCR results were the discharge criteria. Eleven patients recovered well and were discharged in good clinical conditions. One patient who needed mechanical ventilation in the course of treatment was not completely stable and the prognosis seems to be disappointing (Table 3).

In addition to fever for more than 24 hours, simultaneous respiratory and gastrointestinal symptoms were seen in most of the patients, that is in favor of the two-organ involvement. The evaluation of inflammatory markers in future studies is proposed to rule out the possibility of multi system inflammatory syndrome in children related to COVID-19. Small sample size and single center evaluation were our two main limitations. It is worth mentioning that the results of adult studies may not be applicable to children and multicenter larger studies focusing on the hospitalized pediatric population are necessary.

In conclusion, this study was the initial experience of one of the busiest Iranian pediatric centers during COVID19 pandemic. Some of the patients managed easily, but oth- ers had typical findings with no clinical suspicion at the beginning or had prolonged hospital stay, especially patients with significant underlying conditions. Computed tomography appearances also depended on underlying conditions. Our treatment protocols were effective for almost all patients.

\section{Footnotes}

Authors' Contributions: Study concept and design: Fatemeh Zamani and Afshar Ghamari. Analysis and interpretation of data: Afshar Ghamari and Gisoo Darban Hosseini Amirkhiz. Drafting of the manuscript: Afshar Ghamari. Critical revision of the manuscript for important intellectual content: Fatemeh Zamani, Omid Ghaemi, and Neda Pak. Statistical analysis: Afshar Ghamari.

Conflict of Interests: The authors have no conflicts of interest relevant to this article to disclose.

Ethical Approval: This retrospective observational study approved by the Chancellor of Research, Tehran University of Medical Sciences (code: IR.TUMS.VCR.REC.1399.006). 
Funding/Support: This study was done with no specific funding support.

Informed Consent: The informed consent was waived by the Ethics Committee.

\section{References}

1. World Health Organization. WHO Director-General's opening remarks at the media briefing on COVID-19-11 March 2020. Geneva, Switzerland; 2020.

2. Li W, Cui H, Li K, Fang Y, Li S. Chest computed tomography in children with COVID-19 respiratory infection. Pediatr Radiol. 2020;50(6):796-9. doi: 10.1007/s00247-020-04656-7. [PubMed: 32162081]. [PubMed Central: PMC7080075].

3. $\mathrm{Wu} \mathrm{Z,} \mathrm{McGoogan} \mathrm{JM.} \mathrm{Characteristics} \mathrm{of} \mathrm{and} \mathrm{Important} \mathrm{Lessons}$ From the Coronavirus Disease 2019 (COVID-19) Outbreak in China: Summary of a Report of 72314 Cases From the Chinese Center for Disease Control and Prevention. JAMA. 2020;323(13):1239-42. doi 10.1001/jama.2020.2648. [PubMed: 32091533].

4. Cai JH, Wang XS, Ge YL, Xia AM, Chang HL, Tian H, et al. [First case of 2019 novel coronavirus infection in children in Shanghai]. Zhonghua Er Ke Za Zhi. 2020;58(0). Chinese. E002. doi: 10.3760/cma.j.issn.05781310.2020.0002. [PubMed: 32023679].

5. Jiehao C, Jin X, Daojiong L, Zhi Y, Lei X, Zhenghai Q, et al. A Case Series of Children With 2019 Novel Coronavirus Infection: Clinical and Epidemiological Features. Clin Infect Dis. 2020;71(6):154751. doi: 10.1093/cid/ciaa198. [PubMed: 32112072]. [PubMed Central: PMC7108143].

6. Chan JF, Yuan S, Kok K, To KK, Chu H, Yang J, et al. A familial cluster of pneumonia associated with the 2019 novel coronavirus indicating person-to-person transmission: a study of a family cluster. Lancet. 2020;395(10223):514-23. doi:10.1016/s0140-6736(20)30154-9.

7. Park JY, Han MS, Park KU, Kim JY, Choi EH. First Pediatric Case of Coronavirus Disease 2019 in Korea. J Korean Med Sci. 2020;35(11). e124. doi: 10.3346/jkms.2020.35.e124. [PubMed: 32193905]. [PubMed Central: PMC7086086].

8. Rahimzadeh G, Ekrami Noghabi M, Kadkhodaei Elyaderani F, Navaeifar MR, Enayati AA, Manafi Anari A, et al. COVID-19 Infection in Iranian Children: A Case Series of 9 Patients. J Pediatric Rev. 2020;8(2):139-44. doi: 10.32598/jpr.8.2.139.

9. Chen N, Zhou M, Dong X, Qu J, Gong F, Han Y, et al. Epidemiological and clinical characteristics of 99 cases of 2019 novel coronavirus pneumonia in Wuhan, China: a descriptive study. Lancet. 2020;395(10223):507-13. doi: 10.1016/s0140-6736(20)30211-7.

10. Huang C, Wang Y, Li X, Ren L, Zhao J, Hu Y, et al. Clinical features of patients infected with 2019 novel coronavirus in Wuhan, China. Lancet. 2020;395(10223):497-506. doi: 10.1016/S0140-6736(20)30183-5. [PubMed: 31986264]. [PubMed Central: PMC7159299].

11. Wu J, Wu X, Zeng W, Guo D, Fang Z, Chen L, et al. Chest CT Findings in Patients With Coronavirus Disease 2019 and Its Relationship With Clinical Features. Invest Radiol. 2020;55(5):257-61. doi: 10.1097/RLI.0000000000000670. [PubMed:32091414]. [PubMed Central: PMC7147284].

12. Pan F, Ye T, Sun P, Gui S, Liang B, Li L, et al. Time Course of Lung Changes at Chest CT during Recovery from Coronavirus Disease 2019 (COVID19). Radiology. 2020;295(3):715-21. doi: 10.1148/radiol.2020200370. [PubMed: 32053470]. [PubMed Central: PMC7233367].

13. Xia W, Shao J, Guo Y, Peng X, Li Z, Hu D. Clinical and CT features in pediatric patients with COVID-19 infection: Different points from adults. Pediatr Pulmonol. 2020;55(5):1169-74. doi: 10.1002/ppul.24718. [PubMed: 32134205]. [PubMed Central: PMC7168071].

14. Lee EY, McAdam AJ, Chaudry G, Fishman MP, Zurakowski D, Boiselle PM. Swine-origin influenza a (H1N1) viral infection in children: initial chest radiographic findings. Radiology. 2010;254(3):934-41. doi: 10.1148/radiol.09092083. [PubMed: 20032128].

15. Lu X, Zhang L, Du H, Zhang J, Li YY, Qu J, et al. SARS-CoV-2 Infection in Children. N Engl J Med. 2020;382(17):1663-5. doi: 10.1056/NEJMc2005073. [PubMed: 32187458]. [PubMed Central: PMC7121177]. 